\title{
A Proposed Web Based Real Time Brain Computer Interface (BCI) System for Usability Testing
}

\author{
https://doi.org/10.3991/ijoe.v15i07.10447
}

\author{
C. A. Hewawasam Puwakpitiyage ( $\left.{ }^{\bowtie}\right)$, V. R. Paramesura Rao, \\ M. S. A. Muhammad Azizi, W. J. Tee, R. K. Murugesan \\ Taylor's University, Subang Jaya, Malaysia \\ vraga8@yahoo.com \\ M. D. Hamzah \\ Hilti Asia IT Services Sdn. Bhd, Petaling Jaya, Malaysia
}

\begin{abstract}
Hallway testing, Remote Usability testing, Expert review, Automated expert review and A/B testing are the methods commonly used for Usability testing. However, there is no reliable system that integrates Brain Computer Interface (BCI) into the testing process with focus given towards user emotion analysis using electroencephalography (EEG) signals. This paper proposes a system that would be able to identify user emotions while they are conducting usability tests and the results would be able to increase the accuracy of the usability test. In the proposed system the results of the usability test would be displayed in real time on a dashboard and a summary report can be generated for distribution.
\end{abstract}

Keywords - BCI, EEG, usability testing

\section{Introduction}

Brain Computer Interface (BCI) is an area of Computer Science that aims to capture brain activity in the form of electroencephalography (EEG) waves and convert those signals to commands readable by computers using a series of algorithms [1]. Typically, EEG signal analysis is conducted in the medical field to identify patients' suffering from neurological diseases such as epilepsy. With the availability of cheap hardware such as Open BCI [2] and Emotiv Epoch [3], the possibility of integrating $\mathrm{BCI}$ into business applications are vast. A new area that is being explored is the aspect of recognising emotions in real time using EEG waves [4]. This paper proposes a system which integrates real-time emotion recognition to usability testing.

This paper intends to propose a usability testing method which incorporates Brain Computer Interface (BCI) into the testing process to identify user's emotions while using the system that is being tested. The process of conducting usability tests for 
software can be refined by integrating BCI into the process. BCI would be used to identify the user's emotions in real time and the data can be used to improve the outcome of the usability tests conducted. In this paper, we propose a web-based system (dashboard) that would display analysis of user emotions as the usability test is conducted.

The organization of the paper is as follow, Section II will reviews the existing literature on usability testing and BCI, Section III describes the proposed solution that includes the hardware, software and system architecture of the proposed system, and finally, Section IV concludes the paper.

\section{Literature Review on Usability Testing and BCI}

\subsection{Usability testing and current methods}

In the context of human computer interactions, usability is defined as the "extent to which a product can be used by specified users to achieve specified goals with effectiveness, efficiency, and satisfaction in a specified context of use" [3]. In other words, a system can be said to have achieved its usability goals if its intended users are able to use the system as envisioned. Nielsen has identified 5 attributes of usability, namely efficiency, satisfaction, learnability, memorability, and errors rate [5]. A system can be considered usable if it scores a satisfactory level in all these aspects. Usability in software design can also be defined as the degree to which a software can be used by its intended audience for the purpose that the software was designed for while maintaining a high standard of effectiveness, efficiency, and satisfaction [6].

In order to ascertain whether a system is usable, a series of usability tests have to be conducted with real users. Among usability testing methods that are currently being used is heuristic evaluation where usability experts' judge whether the system follows currently established principles and cognitive walkthrough where a detailed list of steps are used to test a user's problem-solving process while using the system [4]. Usability testing, on the other hand, can be defined as a process, which determine how well users are able to use a product through systemic observations.

The sphere of usability testing is large as there are many methods being practiced. Typical methods include hallway testing where randomly selected users are asked to use the system, this helps designers identify major loopholes in the design. Expert review is another method of conducting usability testing. Here experts with experience within the domains test the system to identify its usability.

In addition to the traditional methods discussed above, a research published in 2017 discusses a pattern-based usability testing method [1]. The paper acknowledges that there are some usability test guidelines that can be tested automatically. It suggests different strategies to test these guidelines. Another proposed system describes how usability tests are recorded using paper and how an automated system called Usability Management System (USEMATE) can be used to automate some 
aspects of usability testing while increasing efficiency of each test [4]. The goal of USEMATE is to reduce the costs of conducting usability tests since it is understood that usability testing is one of the most expensive methods of evaluation [7].

As mentioned earlier, heuristic evaluation is one of the common methods of conducting usability tests. A paper on heuristic evaluation suggests that it is one of the most effective methods of conducting usability tests as it uses Nielsen's 10 usability heuristics list to conduct the tests [8]. The research team used public university websites to conduct their experiment by using a set of heuristic evaluation, the good and bad websites were identified.

Another research argues that the Graphical User Interface (GUI) testing is the best way to evaluate the usability of a newly developed system [9]. It is stated that the largest contributors towards the usability of a system are Event Coverage, Ease of Use, and Ease of Understanding. This research recommends a fuzzy logic model to predict the usability of a test case.

The idea of combining BCI and usability testing is relatively new and left unexplored. This paper aims to propose a system to identify user emotions through EEG waves and using this data to support usability testing.

\subsection{Problems with current methods}

Unable to accurately identify a user's emotional state while they are conducting the usability test: The emotional state of a user is an important aspect to consider in order to measure user satisfaction level from the system under test [10]. Inability to know how a user feels accurately can have a negative effect on the results of the test making it inaccurate. Although there are different methods being used to guess the user's state of emotions while using the system, there is no standard method to identify and measure emotions in real time. It is important to differentiate the emotion levels at different stages of the usability test as different stages may trigger different emotions.

Reliability of data collected may not be high: Among techniques used to collect data are surveyed questionnaires, shadowing the user, interviews etc. Although this method may be a good approach when collecting quantitative data, it may not be the best way to identify user satisfaction and emotional state while conducting the usability tests. This is because they would be a possibility of a high level of bias such as picking the inaccurate representation of their emotion when the user is filling up the survey which would result in low accuracy.

\subsection{Brain Computer Interface (BCI)}

According to a research by Vallabhaneni, BCI can be classified as a communication method that depends on brain activity [11]. The brain activity that is also known as neural activity is typically recorded from a subject using two different types of techniques known as invasive and non-invasive. The human brain needs to be explored first to define what neural activity is and what causes them? 
The brain is the most complex organ known to man and it is the centre of the nervous system. In humans, the brain is located in the skull close to other sensory organs such as eyes, ears, and nose. According to research by Pelvig, it is estimated that a normal human brain is made up of 13-33 billion neurons that are interconnected through synapses [12]. A network of neurons connects different parts of the brain to each other. These neurons communicate with each other by passing electrical signals from one neuron to another. The electrical impulses are transferred from one cell to another through synapses. The electrical impulse travels from one cell to another through the release and reception of neurotransmitters into the extracellular space [13]. As a by-product of this activity, an electric field is generated in the brain. If a large enough area of the brain is simultaneously activated, the field generated can be detected outside the scalp. The electric field is recorded using a method known as electroencephalography (EEG) [14].

The electric field can be read using two methods that were mentioned earlier, invasive and non-invasive. The invasive method receives data through electrodes that have been surgically implanted on the surface or inside the brain. This is typically done in a medical condition such as epilepsy where accurate as well as a constant recording of data is required. The type of invasive EEG that sits on the surface of the brain is known as subdural EEG while the type inside the brain is known as depth EEG. The main advantage of using invasive EEG is stronger signals that are recorded due to proximity to the source (brain). The data collected through invasive methods are also referred to as electrocorticography (ECOG).

The non-invasive EEG collection method is where the electrodes are placed on the scalp of the subject and data is read from it. These electrodes are "pasted" onto the scalp using a conductive gel to ease the data collection process. It is also common to use a set of electrodes combined into a cap where the electrodes are embedded, making it easier to use. The main advantage of using the non-invasive method is the fact that there is no permanent effect on the subject. It is a painless temporary process that does not leave any side effects on the subject. However, compared to invasive methods, non-invasive methods would be susceptible to noise, which are distractions on the wave created by external factors such as muscle movement [15].

EEG waves can be divided into 5 main types of waves based on frequency namely Delta, Theta, Alpha, Beta, and Gamma. Delta is from $0 \mathrm{~Hz}$ to $4 \mathrm{~Hz}$ and is usually the highest in amplitude as well as the slowest. Usually seen in adults during sleep and more frequently in babies, Theta waves are waves of frequency from $4 \mathrm{~Hz}$ to $7 \mathrm{~Hz}$. It is usually seen in young children or in adults when they are drowsy and can also be seen during meditation [16]. Abnormal brain activity is usually detected if there is an excess of theta waves from a patient. Alpha, on the other hand, ranges from $7 \mathrm{~Hz}$ to $13 \mathrm{~Hz}$ in frequency [17]. Alpha waves are said to be stronger when eyes are closed, and the subject is relaxed and weakens when eyes are open.

Beta is the frequency range from $14 \mathrm{~Hz}$ to about $30 \mathrm{~Hz}$ and can be detected from both sides of the head. "Beta activity is closely linked to motor behaviour and is generally attenuated during active movements", [18]. In a typical scenario, low amplitude beta waves can be seen during muscle movements and typically more prominent when a subject is active. Lastly, Gamma waves have a frequency range of 
$30 \mathrm{~Hz}-100 \mathrm{~Hz}$ and currently theorised to be the combination of different parts of the neurons of the brain to carry out a complex task such as cognitive or motor oriented.

The EEG data that is collected through the non-invasive method is always corrupted by noise known as artifacts. These artifacts are electrical signals that originate from outside the brain typically due to muscle movements or even eye movements.

Signal pre-processing and removal of noise would have to be conducted in order to remove as many artifacts as possible before analysing the data for other features.

$\mathrm{BCI}$ is an area of science that focuses on creating a communication pathway between the brain and an external device. It is typically understood that in BCI there would be a bidirectional information flow, from the brain to the device and feedback from the device would be obtained through other sensory organs. Early research into BCI has been focused on the medical field such as neuroprosthetics that is commonly used to help patients suffering from hearing disabilities. A hearing BCI device for example would listen to a sound, convert it to a signal and send it directly to the brain. The brain would manage these signals as if from a natural source provided the device was configured properly. Since the 1990s these practices have been commercialized and BCI advancements have directly impacted many lives by improving their quality of life.

The research by Mor states that BCI research is currently being conducted in areas outside of medicine such as security, awareness monitoring, gaming, augmented reality, and even in commerce [19]. This means there is a commercial value of BCI outside of medicine and it could even lead the Web 5.0. It is accepted that the goal of $\mathrm{BCI}$ is not to listen to what a person is thinking rather use thought to conduct an activity or achieve an outcome [20].

\subsection{Feature extraction algorithms used in BCI}

According to Jenke, feature extraction is typically conducted by isolating features in few different domains such as time, frequency, and time-frequency. Features are usually extracted from signals that originate from a single electrode from the EEG headset. However, sometimes signals from multiple headsets can also be combined to form a unified set of signals and features can be extracted from there [21]. An analysis of the three different domains that are used in feature extraction algorithms of EEG waves is given below.

Time domain feature extraction: Time domain feature extraction is said to be the weakest among the three different domains [21]. Although there are few different feature extraction algorithms in the time domain, the most efficient algorithm is said to be High Order Crossing (HOC) algorithm. HOC algorithm focuses on a more robust feature of EEG which is the wave oscillatory pattern of an EEG wave. HOC's use is also validated by another research where it uses this algorithm to extract features related to emotions from EEG [22]. This algorithm would apply a sequence of high-pass filters to an EEG wave.

Frequency domain feature extraction: Frequency domain feature extraction, on the other hand, focusses on different frequency bands that are showcased by EEG 
waves to analyse emotions. There are two common approaches to this method namely Band Power approach and Higher Order Spectra (HOS) approach.

The band power method divides EEG waves into bands of different frequencies. Once the features are extracted, it is ready for feature classification that is also known as feature mapping:

Time-Frequency domain feature extraction: Algorithms of this approach is a lot more powerful compared to the previous two approaches because it is capable of extracting a lot more information from non-stationary waves by taking into account the dynamic changes that the wave goes through. Three common algorithms that use this approach is the Hilbert-Huang Spectrum (HHS), and Discrete Wavelet Transform algorithm.

By using Hilbert-Huang Spectrum (HHS), here meaningful features are extracted by comparing the amplitude of the wave against the phase of the wave. Average of the frequencies can also be used, and the benefits of HHS really depends on the classification algorithm that is used after this stage. Another technique of TimeFrequency feature extraction is the Discrete Wavelet Transformation (DWT) algorithm. This algorithm breaks each wave down to different levels based on frequency ranges while maintaining the time property of each wave. According to research by Murugappan, DWT wave analysis can be used to extract emotions from EEG by using the db4 wavelet function [23].

E Signal Mapping Algorithms used in BCI: In the previous subsection, we have discussed signal feature extraction algorithms. Those algorithms would channel the output to an algorithm for feature mapping or classification. The goal would be to extract the features related to emotions from EEG waves and subsequently map them to a specific emotion through a feature-mapping algorithm.

Support Vector Machine (SVM): One of the commonly used signal classifier is Support Vector Machine (SVM) model [24]. It is a machine learning model which can be used to analyse and classify a set of data. The general method would be to employ a machine learning algorithm to a training data set of a specific emotion for example happiness. Then create a predictive model that has a relatively high level of accuracy. Upon which, the EEG wave that has been extracted would be sent to the predictive model for classification. When an arbitrary level of match is obtained, we can safely assume that the specific emotion was present in the EEG wave. The process can be repeated with other emotions as well to obtain a comprehensive analysis.

Another advantage of taking the SVM approach is its compatibility with the DWT algorithm that we explored in the previous section. It is recommended that a combination of SVM and Hidden Markov Model (HMM) is used to achieve a higher level of accuracy of the predictive model.

Neural Network: A research by Mangalagowri has recommended a neural network based approach for feature classification [25]. At the neural network level of data flow, an algorithm known as "Feed Forward Backward" is used to classify features. A training model using the neural network is created to analyse emotions by focusing on a few electrodes that are connected to the neuroimaging device. 


\section{Proposed Solution}

The proposed system would be a web-based system where the EEG acquisition device (hardware) would be connected to a computer that is capable of constantly uploading the stream of data to the cloud for processing and distribution.

\subsection{Hardware}

Hardware capable of reading and transmitting EEG waves will be used for signal acquisition and transmission. Emotiv EPOC is a device that fits this requirement well. Emotiv EPOC is an EEG acquisition tool by Emotiv that provides up to 14 channels of signals and has a mere 3-5 minute set up time. This would allow usability tests to be conducted with relative ease and reduce time wasted on setting up. In addition to that, the data transmission is wireless and Emotiv EPOC is battery powered. This means the test set up would not be cluttered with wires thus reducing possible data inaccuracies due to incorrect setting up. Fig 1 below shows the top view of the Emotiv EPOC device.

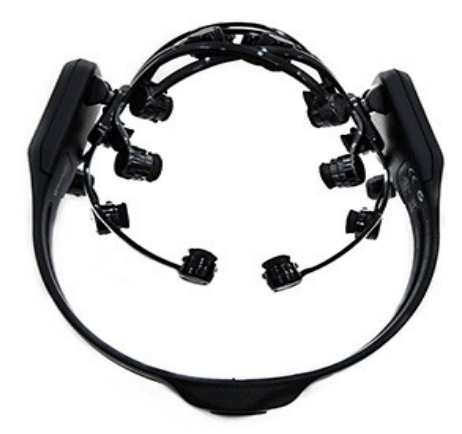

Fig. 1. Top view of Emotiv EPOC device

The Emotiv EPOC also comes with a series of Application Programming Interfaces (APIs) that can be used for analysis. The Raw EEG API will be used by the proposed system to obtain data from the devices which will then be used for analysis. The languages which will be used to develop the analytical tools will be discussed in the next section.

\subsection{Web development frameworks - Angular JS}

AngularJS is a JavaScript based front-end framework that can be used to develop single page applications as well as multi-page web applications [26]. It was developed by Google and is maintained by them and the community of developers. AngularJS also extends from Hypertext Markup Language (HTML) which makes it easy to read and easier to pick up compared to other frameworks [27]. 
The main advantage of using AngularJS is the fact that applications built using this framework would be using the Model-View-Controller (MVC). MVC is a software design pattern where the application is separated into three main logical components: the model, the view, and the controller. Latest AngularJS versions use a hybrid version of the traditional MVC where it uses the Model-View-ViewModel (MVVM) design pattern [28]. The model in the design pattern refers to the data. Since all data types are treated as objects in JavaScript, the model would consist of all the data objects. In AngularJS, the view is the HTML that exists after AngularJS has parsed and compiled. It also includes all the rendered mark-ups as well as data bindings. The ViewModel, on the other hand, performs a hybrid of the controller function where it provides views with their data as well as methods to alter the data.

The main disadvantage of using AngularJS would be its performance, especially when handling a large set of data. The system would constantly be updated with a large inflow of data such as raw EEG as well as the inflow of processed data. Since there is no virtual Document Object Model (DOM) to track changes and only display the updates, the regular DOM would consistently be injected with updates causing performance delays when handling a large dataset [29].

In conclusion, AngularJS may be a good choice since it is also part of the MEAN stack which is used to develop full-stack web applications [30]. MEAN stack is made up of MongoDB which is a NoSQL database, Express.JS which is a web application framework that works in the backend communicating with the database and the frontend environment, AngularJS and Node.js which is a server-side environment built using JavaScript. However, performance concerns are there and the dashboard requiring constant updates with live data streams may need a framework that is capable of high-performance rendering.

\subsection{BCI usability testing system}

The architectural overview of the system is illustrated in Fig. 2. Types of users involved:

Test subject user: The individual that would be using the system under test while wearing the EEG acquisition device. When selecting this individual, special attention should be given to ensuring the person has a relatively normal medical history and has not been diagnosed with any condition that may cause abnormality in their EEG signals. An emotion inducing video should be shown beforehand to create a baseline data set unique to each user. This data set would be used for signal classification at the cloud layer later on.

Test supervisor: The individual that conducts the tests and observe the dashboard. Since it is a cloud-based system, other stakeholders can view the results remotely as well. The test supervisor would also be in charge of assisting the setting up of hardware and ensuring all nodes are connected and are active. 


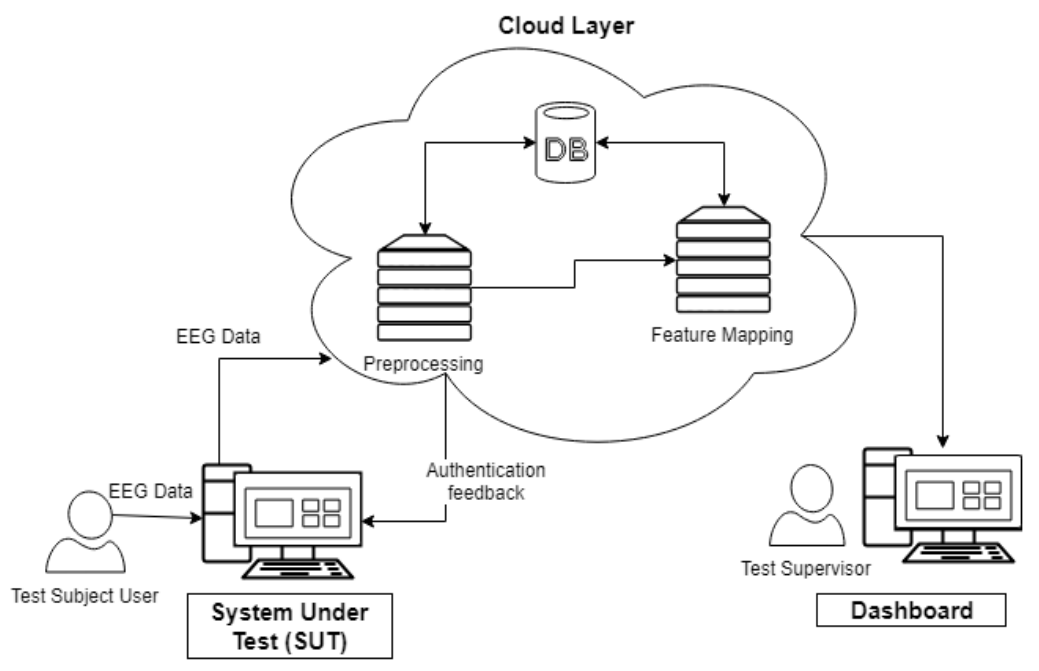

Fig. 2. High-Level Architecture of the Proposed System

The system can be divided into three modules which are the System Under Test (SUT), Cloud Layer and Dashboard. System Under Test (SUT) module is the system that is going through the usability testing process. It can be any computer-based system ranging from simple websites to complex web applications. SUT can also be two systems that the Test Supervisor wants to test as an A/B test. Data acquisition and transmission would also happen at this layer as depicted in Fig. 2.

Cloud layer module is where the analysis of EEG signals would take place. A database is used in the cloud to store credentials and other data. At this layer, the signal stream would go through a series of algorithms which will handle preprocessing (removal of noise) and feature mapping (extraction of features from EEG signals and mapping them to known emotions). There are three different approaches to conduct feature extraction namely time domain, frequency domain, and timefrequency domain approach. Each of this approach has numerous algorithms that can be used. As a feature extraction algorithm, we would recommend Discrete Wavelet Transformation (DWT) algorithm which is based on the time-frequency approach because it is capable of time-frequency localization, multiscale zooming, and noise filtering [24].

The output would then be channelled to an algorithm for feature mapping or classification. The goal would be to extract the features related to emotions from EEG waves and subsequently map them to a specific emotion through a feature mapping algorithm. One of the commonly used signal classifiers is Support Vector Machine (SVM) model [24]. It is a machine learning model which can be used to analyse and classify a set of data. The general method would be to employ a machine learning algorithm with a training data set of a specific emotion for example happiness. Then create a predictive model that has a relatively high level of accuracy. Upon which, the EEG wave that has been extracted would be sent to the predictive model for 
classification and an arbitrary level of match is obtained. We can safely assume that the specific emotion was present in the EEG wave. The process can be repeated with other emotions as well to obtain a comprehensive analysis.

Another advantage of taking the SVM approach is its compatibility with the DWT algorithm [24]. It is recommended that a combination of SVM and Hidden Markov Model (HMM) is used to achieve a higher level of accuracy of the predictive model [31]. These models can be created using cloud service platforms such as Amazon Web Services and Azure.

The third module is the Dashboard. This module is where all the data from the cloud server is displayed in a comprehensible format. The dashboard would have the functionalities such as general view, specific data stream analysis, generate a report for the session as well as download or share report. The emotions that the Test Subject User felt would be shown using graphs and at the end of the session, the aggregated results can also be shown. The Test Supervisor can also view previous results and identify whether the usability levels have increased if the same system is retested. The dashboard would also have the option to generate a report of each session which allows the Test Supervisor to download a report containing a summary of the session.

\section{Conclusion}

This paper proposed a web-based system that can be used for usability testing that incorporates BCI into the testing process through emotion recognition. The proposed system aims to improve the outcome of a usability test by giving the testing team the opportunity to know user's emotions while they are conducting the usability test. The paper described the architecture of the proposed system as well as recommended tools that should be used with the system. In the future, this research will focus on testing the system and connecting it to other hardware such as eye-tracker which aims to increase the accuracy of the results that can be obtained. The goal would be to use the proposed system to test any computer-based system out there or any that is currently being developed.

\section{$5 \quad$ References}

[1] F. Dias and A. C. R. Paiva, "Pattern-Based Usability Testing," in 2017 IEEE International Conference on Software Testing, Verification and Validation Workshops (ICSTW). 2017, pp. 366-371. https://doi.org/10.1109/ICSTW.2017.65

[2] "OpenBCI - Open Source Biosensing Tools (EEG, EMG, EKG, and more)." [Online]. Available: https://openbci.com/. [Accessed: 18-Nov-2018].

[3] "EMOTIV EPOC+ - 14 Channel Wireless EEG Headset." [Online]. Available: https://www.emotiv.com/epoc/. [Accessed: 18-Nov-2018].

[4] W. F. W. Ahmad, S. Sulaiman, and F. S. Johari, "Usability Management System (USEMATE): A web-based automated system for managing usability testing systematically," in 2010 International Conference on User Science and Engineering (iUSEr), 2010, pp. 110-115. https://doi.org/10.1109/IUSER.2010.5716734 
[5] R. Harrison, D. Flood, and D. Duce, "Usability of mobile applications: literature review and rationale for a new usability model,” J. Interact. Sci., vol. 1, no. 1, pp. 1-16, 2013. https://doi.org/10.1186/2194-0827-1-1

[6] "ISO 9241-11:1998 - Ergonomic requirements for office work with visual display terminals (VDTs) -- Part 11: Guidance on usability." [Online]. Available: https://www.iso.org/standard/16883.html. [Accessed: 03-Oct-2018].

[7] G. Lindgaard and J. Chattratichart, "Usability testing," in Proceedings of the SIGCHI conference on Human factors in computing systems - CHI '07, 2007, p. 1415-1424. https://doi.org/10.1145/1240624.1240839

[8] A. Lodhi, "Usability Heuristics as an assessment parameter: For performing Usability Testing," in 2010 2nd International Conference on Software Technology and Engineering, 2010. https://doi.org/10.1109/ICSTE.2010.5608809

[9] T. Kushwaha and O. P. Sangwan, "Prediction of Usability Level of Test Cases for GUI Based Application Using Fuzzy Logic," in Confluence 2013: The Next Generation Information Technology Summit (4th International Conference), 2013, p. 2.06-2.06. https://doi.org/10.1049/cp.2013.2297

[10] V. do Amaral, L. A. Ferreira, P. T. Aquino, and M. C. F. de Castro, "EEG signal classification in usability experiments," in 2013 ISSNIP Biosignals and Biorobotics Conference: Biosignals and Robotics for Better and Safer Living (BRC), 2013, pp. 1-5.

[11] A. Vallabhaneni, T. Wang, and B. He, "Brain-Computer Interface," in Neural Engineering, Boston, MA: Springer US, 2005, pp. 85-121.

[12] D. P. Pelvig, H. Pakkenberg, A. K. Stark, and B. Pakkenberg, "Neocortical glial cell numbers in human brains," Neurobiol. Aging, vol. 29, no. 11, pp. 1754-1762, Nov. 2008. https://doi.org/10.1016/j.neurobiolaging.2007.04.013

[13] E. S. Bucher and R. M. Wightman, "Electrochemical Analysis of Neurotransmitters," Annu. Rev. Anal. Chem., vol. 8, no. 1, pp. 239-261, Jul. 2015. https://doi.org/10.1146/annurev-anchem-071114-040426

[14] S. Bandyopadhyay, M. Z. Koubeissi, and N. J. Azar, "Physiologic Basis of EEG and Epilepsy," in Epilepsy Board Review, New York, NY: Springer New York, 2017, pp. 313. https://doi.org/10.1007/978-1-4939-6774-2 1

[15] G. L. Gebber, S. Zhong, C. Lewis, and S. M. Barman, "Human brain alpha rhythm: nonlinear oscillation or filtered noise?," Brain Res., vol. 818, no. 2, pp. 556-560, Feb. 1999. https://doi.org/10.1016/S0006-8993(98)01303-1

[16] B. R. Cahn and J. Polich, "Meditation states and traits: EEG, ERP, and neuroimaging studies.," Psychol. Bull., vol. 132, no. 2, pp. 180-211, 2006. https://doi.org/10.1037/0033$\underline{2909.132 .2 .180}$

[17] P. Gerrard and R. Malcolm, "Mechanisms of modafinil: A review of current research.," Neuropsychiatr. Dis. Treat., vol. 3, no. 3, pp. 349-64, Jun. 2007.

[18] G. Pfurtscheller and F. H. Lopes da Silva, "Event-related EEG/MEG synchronization and desynchronization: basic principles.” Clin. Neurophysiol., vol. 110, no. 11, pp. 1842-1857, Nov. 1999. https://doi.org/10.1016/S1388-2457(99)00141-8

[19] M. Mor and L. Juvvala, "Brain Computer Interface," 2014.

[20] J. R. Wolpaw, N. Birbaumer, D. J. Mcfarland, G. Pfurtscheller, and T. M. Vaughan, "Brain-computer interfaces for communication and control," 2002.

[21] R. Jenke, A. Peer, and M. Buss, "Feature Extraction and Selection for Emotion Recognition from EEG,” IEEE Trans. Affect. Comput., vol. 5, no. 3, pp. 327-339, Jul. 2014. https://doi.org/10.1109/TAFFC.2014.2339834

[22] P. Ackermann, C. Kohlschein, J. A. Bitsch, K. Wehrle, and S. Jeschke, "EEG-based automatic emotion recognition: Feature extraction, selection and classification methods," 
in 2016 IEEE 18th International Conference on e-Health Networking, Applications and Services (Healthcom), 2016, pp. 1-6. https://doi.org/10.1109/HealthCom.2016.7749447

[23] M. Murugappan, N. Ramachandran, and Y. Sazali, "Classification of human emotion from EEG using discrete wavelet transform,” J. Biomed. Sci. Eng., vol. 03, no. 04, pp. 390-396, 2010. https://doi.org/10.4236/jbise.2010.34054

[24] K. Guo, H. Candra, H. Yu, H. Li, H. T. Nguyen, and S. W. Su, "EEG-based emotion classification using innovative features and combined SVM and HMM classifier," in 2017 39th Annual International Conference of the IEEE Engineering in Medicine and Biology Society (EMBC), 2017, pp. 489-492. https://doi.org/10.1109/EMBC.2017.8036868

[25] S. G. Mangalagowri and P. C. P. Raj, "EEG feature extraction and classification using feed forward backpropogation algorithm for emotion detection," in 2016 International Conference on Electrical, Electronics, Communication, Computer and Optimization Techniques (ICEECCOT), 2016, pp. 183-187. https://doi.org/10.1109/ICEECCOT.2016.7955211

[26] “Angular.” [Online]. Available: https://angular.io/. [Accessed: 18-Nov-2018].

[27] P. Kozlowski and P. B. Darwin, Mastering web application development with AngularJS. Packt Pub, 2013.

[28] N. Jain, P. Mangal, and D. Mehta, "AngularJS: A Modern MVC Framework in JavaScript,” J. Glob. Res. Comput. Sci., vol. 5, no. 12, 2014.

[29] M. Selakovic and M. Pradel, "Performance issues and optimizations in JavaScript," in Proceedings of the 38th International Conference on Software Engineering - ICSE '16, 2016, pp. 61-72. https://doi.org/10.1145/2884781.2884829

[30] "home - Mongo Express Angular Node." [Online]. Available: http://mean.io/. [Accessed: 18-Nov-2018].

[31] H. Zhu, Y. Xin, and F. Wang, "A novel framework for anomaly detection based on hybrid HMM-SVM model," in 2011 4th IEEE International Conference on Broadband Network and Multimedia Technology, 2011, pp. 670-674. https://doi.org/10.11 $\underline{\text { 09/ICBNMT.2011.6156020 }}$

\section{Authors}

Hewawasam Puwakpitiyage Chamode Anjana received his Bachelor of Computer Science (Hons) from Taylor's University in 2019. His area of interest includes Cloud Computing, Mobile Applications Development and Artificial Intelligence.

Vegnish Rao Paramesura Rao graduated with the Bachelor of Compute Science (Hons) from Taylor's University in 2019. His area of interests include IoT, Machine Learning, Cloud Computing and Software Engineering.

Muhammad Syahir Amali bin Muhammad Azizi is a recent graduate from Taylor's University whom had majored Computer Security and Forensics in 2019. His area of interests include artificial intelligence, big data, and game design

Wee Jing Tee is currently the senior lecturer from Taylor's University School of Computing and IT. He graduated with Master of Computer Science from University Malaya. He has 17 years of working experience with 12 years in teaching and 6 years in ICT industry. His research interests are in blockchain, artificial intelligent, and brain computer interface. He has certifications in CPRE, CTFL and TRIZ. 
Dr Raja Kumar Murugesan is an Associate Professor of Computer Science, and Head of Research for the Faculty of Innovation and Technology at Taylor's University, Malaysia. He has a $\mathrm{PhD}$ in Advanced Computer Networks from the Universiti Sains Malaysia, and has over 28 years' experience as an educator. His research interests include IPv6, and Future Internet, Internet Governance, Computer Networks, Network Security, IoT, Blockchain, Machine Learning, and Affective Computing. He is a member of the IEEE and IEEE Communications Society, Internet Society (ISOC), and associated with the IPv6 Forum, Asia Pacific Advanced Network Group (APAN), Internet2, and Malaysia Network Operator Group (MyNOG) member's community.

M. D. Hamzah received his Ph.D. (Computer Engineering) from The University of Electro-Communications, Tokyo, Japan in 2009. Previously he worked as a senior researcher and test lead in Brother Industries Ltd., Japan and now working as a senior application test engineer in Hilti Asia IT Services, Malaysia. His research interests are in the field of the intelligent user interface, cognitive engineering, software testing and mobile computing.

Article submitted 2019-01-10. Resubmitted 2019-03-23. Final acceptance 2019-03-26. Final version published as submitted by the authors. 